\title{
Introduction to Time and Families
}

\author{
Martin Covey, CFLE
}

\begin{abstract}
This issue of Michigan Family Review has a focus on the interface of time and families. The articles in this issue address time and families from different perspectives but they have a common element that sets this issue apart from other writings about families and time. One article explores the juxtaposition of families in regards to increasing demands in the roles of individual family members. Another article proposes a research instrument designed to explore the value family time has for older adults. In the case of both articles, the issue of feelings that family members have regarding their experience of family time is examined.
\end{abstract}

Key words: families, time, measurement

In the preface to his book, Families \& Time, Daly (2001) reflected on his work with young fathers and their children. From his research, he observed that these fathers often lamented about the scarcity of time with their children, wondering how they could spend more time with their children, and how they could manage to make more time for their children. He states, "Time appeared as a dominant currency in their experience of family: It was the measure of value in their relationship with their children" (Italics inserted).

From this foundational statement, Daly explores the issue of time, analyzing the "conceptual meanings of time for families" (2001, p. xii). He continues to investigate the complexities of time as viewed by different generations, even within the same family, His analysis brings to light the observation that there is "a set of underlying structural constraints that may be operating" (p. 293) when it comes to family time. Family time becomes problematic when the disparate views of family time are not recognized and accommodated from both research perspective and from the perspective of individual families. Later in the book, Daly observes that "when the discordant nature of family time is seen as a contradiction, it shifts attention from seeing family time as a personal trouble that requires a private solution, to a pervasive systemic dilemma that challenges all families in some way" (Daly, 2001, p. 293). It would seem then that interventions for families designed to assist in the difficult task of time management would necessarily take place in both the micro and macro levels.

A macro system approach for the family professional would be working to modify the "pervasive systemic dilemmas" (Daly, 2001, p. 293) that exacerbate the negative feelings ubiquitously experienced by families. He explains, 
The dominant cultural response is for parents to at least think about working less, even though few seem to be able to follow through on this. However, if parents are unable to change the nature of their demands in order to come closer to the ideal, then it leads one to question whether the ideals themselves need to be opened to reexamination. These are questions at a cultural and ideological level that open to question the dominant discourse of family time. (pp. 293-294)

One such "ideal" can be described as the "hurried child" syndrome. The syndrome results when parents over-schedule their children with too many activities, in the well meaning attempt to provide opportunities to enhance their development (Lamanna \& Riedmann, 2009, p. 280). Not only do the over-scheduled children suffer the stress of a schedule that is too hectic, the parents become harried as well from the subsequent stress of the overlap of their hectic work environments and the necessity of keeping up with their children's busy schedules.

The resulting time strain is an example of a systemic dilemma that communicates to parents that to be good parents, their children be active in a multiplicity of activities at all times. Their dilemma is the desire to be "good parents," but not having the emotional resources or the time resources to carry out what appears to be a cultural imperative. An example of a macro system approach addressing this dilemma is the "Family Life $1^{\text {st" }}$ movement (Doherty \& Boyte). The movement mobilized a broad range of community partners (parents, clergy, teachers, community activists, and other professionals who were interested in

reversing the deleterious effects of the consumer culture of childhood, in which parents see themselves as competitive providers of services to children, and children are overscheduled in a frantic pursuit of experiences and opportunities for personal enrichment and advantage over peers. (no page number).

Private solutions might be pragmatic discussions involving methods for scheduling, prioritizing, and managing individual and family schedules and activities that impinge on family time. Other private solutions might be more affective in nature - solutions helping individuals and families learn different ways of viewing and maximizing time for their families. For example, Kantor and Lehr (2003) observe that a family's orientation to time "works as a filter for members' experience in the present." (p.78). If one or more members of a family are past oriented, and other family members are future oriented, it stands that the family together would experience dissonance in the way they function in regards to time. A meaningful intervention for the family might be assisting in integrating the ways they view time. Initiating this discussion in the family may lead to new discoveries of values underlying the decisions the family makes in Michigan Family Review, 13, 1-4, 2009 (C) Michigan Council on Family Relations 
their choice of activities to participate in or to avoid. Assisting families in the exploration and identification of the values they hold as a family could also help them in goal setting, in resource management, and in filtering out actions, attitudes and activities that do not promote the living out of those values (Covey, 1997).

Daly's (2001) research of family time indicated that two keys in the positive assessment of family time were 1) the notion of togetherness was present, and 2) that the experience was viewed as positive. In a similar sense, the articles contained in this edition of Michigan Family Review contribute a different perspective of how time is viewed by families. In both of the articles it is the feelings toward and about family time that is experienced by families and their members that receive attention.

In their article, Bulanda and Lippman document the conundrum contemporary families face, trying to align increased expectations of the work force with the increased expectations of what is required to be a good parent. The authors acknowledge the contributions of Daly (2001) in regards to the conceptualization of family time, but they suggest that further research should focus on parental feelings regarding the time that is actually spent with their children.

The article by Peters and Bolkan integrates Daly's (2001) notion that different generations within a family see family time differently, with Bulanda and Lippman's observation that a focus for future family time research should incorporate the variable of family members' feelings regarding time spent with the family. The authors propose a survey instrument, the purpose of which is to explore how older adults value shared time with family. Of particular interest are the measures of feelings older adults have regarding family time, both in the present and in the past.

It is exciting to see family research moving forward in the exploration of complex dimensions of the ubiquitous experience of family time. The progress that moves family science from the recognition of the importance of family time, to the diverse conceptualizations of family time, to the exploration of feelings and emotions related to family time goes apace. Hopefully this progress will eventually be reflected in macrosystem and microsystem interventions family professionals have with families. This type of progress would certainly be seen - if only we had more time.... 
References:

Covey, S.R. (1997). The 7 habits of highly effective families. London: Simon \& Schuster.

Daly, K. (2001). Deconstructing family time: From ideology to lived experience. Family Relations, 63, 283-294.

Doherty, W. J., \& Boyte, H. (no date). Family life $1^{\text {st }}$ : Activating a grassroots parents movement. Retrieved June 10, 2009 from: http://www.familyimpactseminars.org/s_wifis13c03.pdf.

Kantor, D. \& Lehr, W. (2003). Inside the family. Cambridge, MA: Meredith Winter.

Lamanna, M.A., \& Riedmann, A. (2009). Marriages and families: Making choices in a diverse society. Belmont, CA: Thomson. 